\title{
Venn Diagrams with Few Vertices
}

\author{
Bette Bultena and Frank Ruskey \\ abultena@csr.csc.uvic.ca, fruskey@csr.csc.uvic.ca \\ Department of Computer Science \\ University of Victoria \\ Victoria, B.C. V8W 3P6, Canada
}

Submitted: September 15, 1998, Accepted: October 1, 1998

\begin{abstract}
An $n$-Venn diagram is a collection of $n$ finitely-intersecting simple closed curves in the plane, such that each of the $2^{n}$ sets $X_{1} \cap X_{2} \cap \cdots \cap X_{n}$, where each $X_{i}$ is the open interior or exterior of the $i$-th curve, is a non-empty connected region. The weight of a region is the number of curves that contain it. A region of weight $k$ is a $k$-region. A monotone Venn diagram with $n$ curves has the property that every $k$-region, where $0<k<n$, is adjacent to at least one $(k-1)$-region and at least one $(k+1)$-region. Monotone diagrams are precisely those that can be drawn with all curves convex.

An $n$-Venn diagram can be interpreted as a planar graph in which the intersection points of the curves are the vertices. For general Venn diagrams, the number of vertices is at least $\left\lceil\frac{2^{n}-2}{n-1}\right\rceil$. Examples are given that demonstrate that this bound can be attained for $1<n \leq 7$. We show that each monotone Venn diagram has at least $\left(\begin{array}{c}n \\ \lfloor n / 2\rfloor\end{array}\right)$ vertices, and that this lower bound can be attained for all $n>1$.
\end{abstract}

Keywords: Venn diagram, dual graph, convex curve, Catalan number.

AMS Classification (primary, secondary): 05C10, 52C99.

\section{Introduction}

There has been a renewed interest in Venn diagrams in the past couple of years. Recent surveys have been written by Ruskey [10] and Hamburger [8]. In this paper we tackle a natural problem that has not received any attention: What is the least number of vertices in a Venn diagram of $n$ curves? Figure 1(a) shows the classic Venn diagram of 3 curves, which contains 6 vertices. The Venn diagram of Figure 1(b) is also constructed with 3 curves, but has only 3 vertices. This second diagram has the minimum number of vertices among all Venn diagrams of 3 curves (a complete listing may be found in Chilakamarri, Hamburger, and Pippert [3]). We show that this is the minimum value in Theorem 2.1 in the following section. 


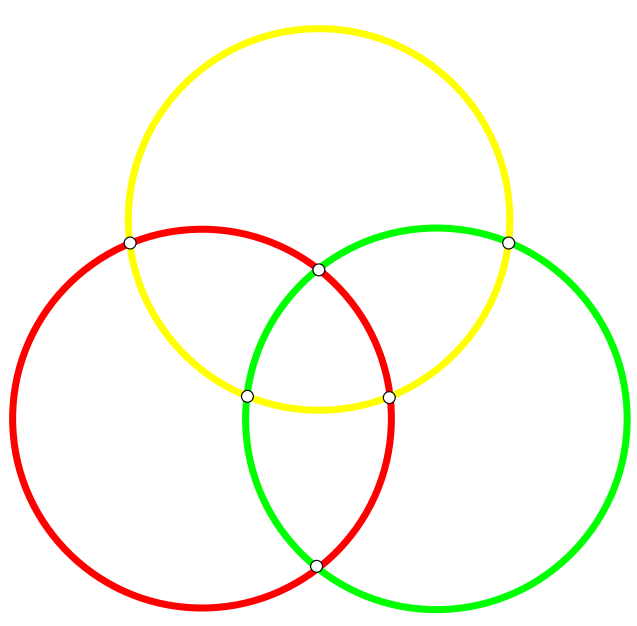

(a) Venn Diagram with 3 curves and 6 vertices

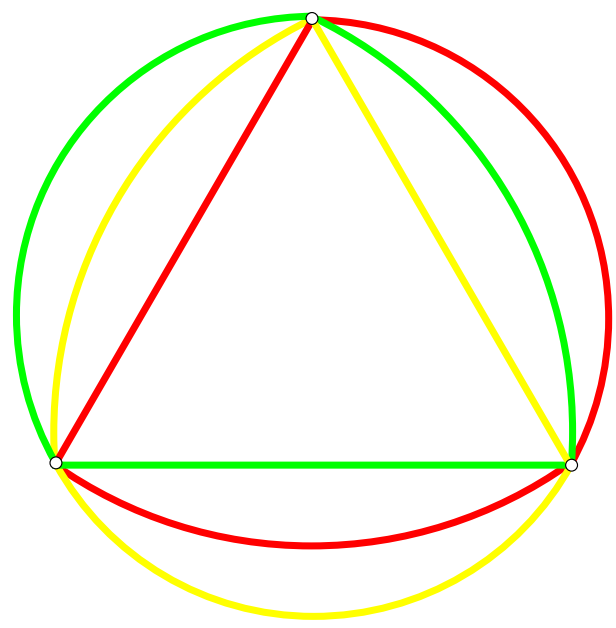

(b) Venn Diagram with 3 curves and 3 vertices

Figure 1: Example of a simple and a non-simple 3-Venn diagram.

We give the relevant graph theoretic definitions in the remainder of this section. Section 2 provides a proof of the lower bound for the number of vertices of general Venn diagrams and provides examples of Venn diagrams that have this minimum number if $1<n \leq 7$. Finding a minimum vertex Venn diagram for $n>7$ remains an open problem. In Section 3, we demonstrate that the upper bound of $\left(\begin{array}{c}n \\ n / 2\end{array}\right)$ for the minimum number of vertices of a monotone Venn diagrams is attainable for all $n>1$. This is demonstrated, using a specific and recursively constructed sequence of diagrams. The proof that the number of vertices is as stated involves the Catalan numbers.

\subsection{Venn Diagrams and Graphs}

Let us review Grünbaum's definition of a Venn diagram [7]. An $n$-Venn diagram in the plane is a collection of simple closed Jordan curves $\mathbf{C}=C_{1}, C_{2}, \ldots, C_{n}$, such that each of the $2^{n}$ sets $X_{1} \cap X_{2} \cap \ldots \cap X_{n}$ is a nonempty and connected region. Each $X_{i}$ is either the bounded interior or the unbounded exterior of $C_{i}$, and this intersection can be uniquely identified by a subset of $\{1,2, \ldots, n\}$, indicating the subset of the indices of the curves whose interiors are included in the intersection. To this definition we add the condition that pairs of curves can intersect only at a finite number of points.

We say that two Venn diagrams are isomorphic if, by continuous transformation of the plane, one of them can be changed into the other or its mirror image [10].

When analyzing a Venn diagram, we often think of it as a plane graph $V$, whose vertices (called Venn vertices) are the intersection points of the curves. The labelled edges of $V$ are of the form $C(v, w)$, where there is a segment on curve $C$ with intersection points $v$ and $w$, and no intersection points between them on $C$. The label of the edge is $i$ if $C=C_{i}$. Each face, including the outer infinite face, is called a region 

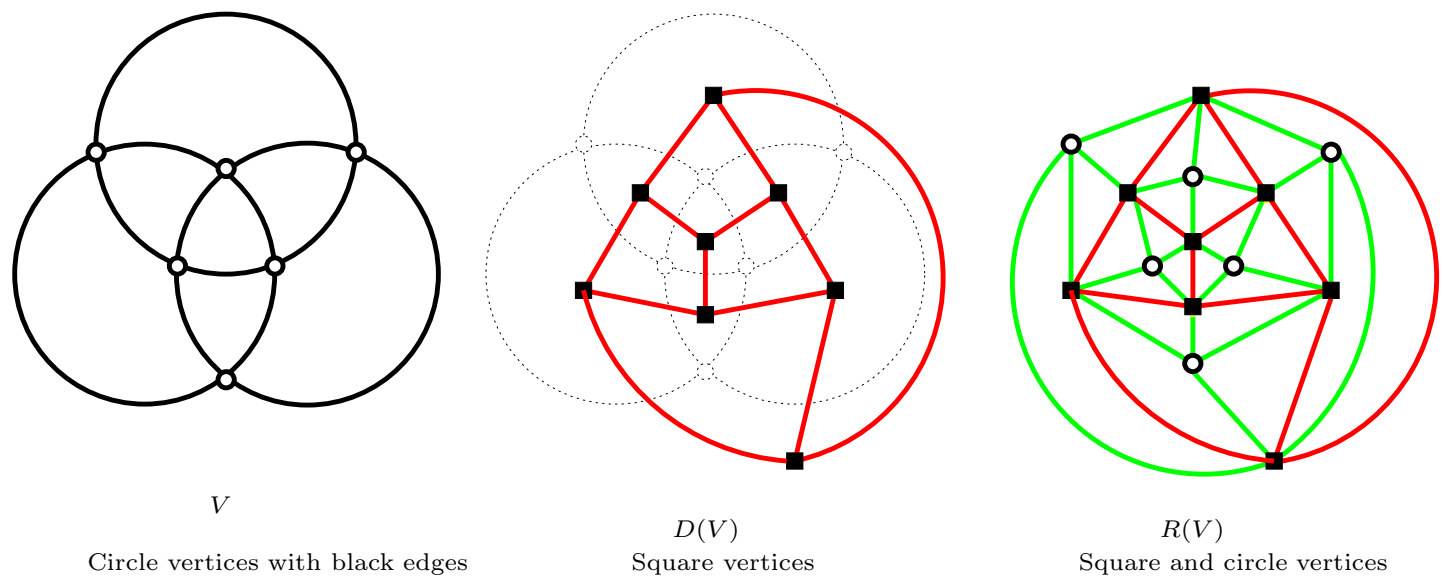

Figure 2: The radual graph construction.

when referring to $V$. Each region in the Venn diagram has associated with it a unique subset of $1,2, \ldots, n$, and a weight. The weight is the number of curves that contain the region and is equal to the cardinality of its representative subset. A region of weight $k$ is referred to as a $k$-region.

A facial walk of a region is a walk taken around the region in clockwise order, recording the edges and vertices bordering the region as they are encountered. It is easy to prove that the graph $V$ is 2-connected, and hence each edge borders exactly two regions. Both vertices of this edge are found on facial walks of both regions. A vertex traversal of a vertex $v$ in a Venn diagram is a circular sequence $C_{0}, C_{1}, \ldots, C_{m}$ of the curves adjacent to $v$, when read in a clockwise rotation around $v$ [10].

We also use the familiar dual graph, $D(V)$, of the Venn diagram. It is constructed by placing a vertex within each region of $V$. For each edge of $V$, a dual graph edge is drawn which connects the vertices within the two adjacent regions. Note that each of the dual vertices corresponds to a face in $V$, and each of the Venn vertices corresponds to a face in $D(V)$. We identify each of the dual vertices by the same subset and weight of the associated region on $V$. We define the directed dual graph, $\vec{D}(V)$, by imposing a direction on each edge so that it is directed from the vertex of larger weight to the vertex of smaller weight [10].

The vertex set of the radual graph $R(V)$ consists of the union of the vertex sets of $V$ and $D(V)$. The edge set of $R(V)$ consists of all edges in $D(V)$ together with edges between each dual vertex and the following specified Venn vertices: In the radual graph, a dual vertex $d$ is adjacent to a Venn vertex $v$ if $v$ is encountered on a facial walk around the region of $V$ containing $d$. The radual graph construction is illustrated in Figure 2. The radual graph of any 2-connected planar graph is itself planar. Note that the edges incident with $d$ in $R(V)$ are alternately incident with Venn vertices and dual vertices as we circle around $d$ in a fixed direction. 


\subsection{Monotone Venn Diagrams}

In this paper we are primarily interested in those Venn diagrams that are monotone. Following [10], we define a diagram to be monotone if and only if the directed dual graph $\vec{D}(V)$ has a unique sink (a vertex with no out-going edges), and a unique source (a vertex with no incoming edges). An equivalent definition of a monotone Venn diagram is that each dual vertex with weight $0<k<n$ in the dual graph is adjacent to a dual vertex with weight $k-1$ and a dual vertex with weight $k+1$.

Monotone diagrams are a natural and interesting class of Venn diagrams. The general constructions of Edwards [5], [6] are monotone. The "necklace property" mentioned in Edwards [4] is a consequence of monotonicity. A Venn diagram is convex if its curves are convex. The Venn diagrams in Figure 1 are both convex. In [1], it is proven that a Venn diagram is isomorphic to a convex Venn diagram if and only if it is monotone. Thus the geometric condition of convexity is equivalent to the purely combinatorial condition of monotonicity.

\section{General Venn Diagrams}

Let $\operatorname{Min}(n)$ be the least number of vertices of a Venn diagram of $n$ curves.

Theorem 2.1 If $n>1$, then

$$
\operatorname{Min}(n) \geq\left\lceil\frac{2^{n}-2}{n-1}\right\rceil \text {. }
$$

Proof: Consider a $n$-Venn diagram $V$, with vertex set $W$. Let $f, v$, and $e$ denote the number of faces, vertices and edges of $V$. We denote the degree of vertex $w$ as $\operatorname{deg}(w)$. By definition, for $w \in W, \operatorname{deg}(w)$ is no more than $2 n$. So

$$
2 n v \geq \sum_{w \in W} \operatorname{deg}(w)=2 e .
$$

By Euler's relation, $e=2^{n}+v-2$, and therefore

$$
v \geq \frac{2^{n}-2}{n-1}
$$

We provide examples of general $n$-Venn diagrams that attain this lower bound for $1<n \leq 7$. Figure 3 shows a minimum 4 -Venn discovered in collaboration with Peter Hamburger. Figure 4 and 5 are diagrams which are successively extended from the minimum 4-Venn diagram, discovered by the first author.

Figure 6 is a polar symmetric minimum 7-Venn diagram, discovered in collaboration with Stirling Chow using a computer search. Note that each vertex has the maximum degree; every curve passes through every vertex. The diagram is symmetric in the sense that each curve of the diagram can be obtained by rotating a given curve 


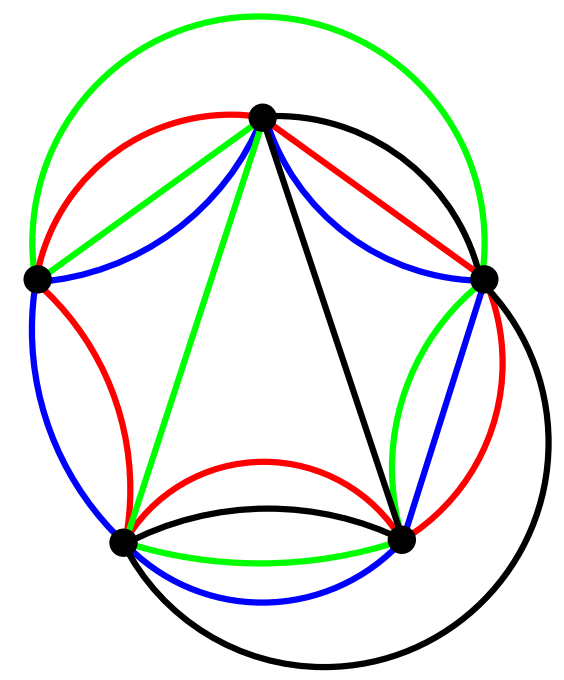

Figure 3: A 4-Venn diagram with 5 vertices.

(e.g., the highlighted one in the diagram) by a multiple of $2 \pi / 7$ about some point on the plane. Symmetric diagrams in this sense can only exist if $n$ is prime. Thus a minimum vertex symmetric diagram might only exist if $n$ is a prime for which $n-1$ divides $2^{n}-2$. The only such primes, $7<n<100$, are 19 and 43 .

The diagrams of this section inspire the conjecture that the lower bound of Theorem 2.1 can be achieved for all numbers $n$. We leave this as an open problem.

\section{Monotone Venn Diagrams}

The following lemmas deal with general plane graphs, illustrating that each dual vertex in the radual graph is bordered by a specific type of cycle. The lemmas are used to prove the lower bound for monotone Venn diagrams.

Lemma 3.1 The degree of a dual vertex $d$ in the radual graph is equal to twice the number of edges on the facial walk of the region containing $d$ in the original plane graph.

Proof: Consider $P, D(P)$, and $R(P)$, a plane graph, its dual graph, and its radual graph, respectively: Let $d$ be a dual vertex within face $F$ of $P$. There are an equal number of edges and vertices on the facial cycle of $F$. Each vertex $v_{i}$ on this cycle is adjacent to $d$ by definition of $R(P)$. Each edge on the facial cycle of $F$ corresponds to an edge between $d$ and another dual vertex $d_{i}$ in region $S$ of $P$. Therefore $d$ is adjacent to the total number of vertices and edges on $F$ 's facial cycle.

Lemma 3.2 The subgraph of the radual graph $R(P)$ induced by the open neighbourhood of a dual vertex $d$ is an alternating cycle of dual vertices and vertices of the plane graph $P$. 


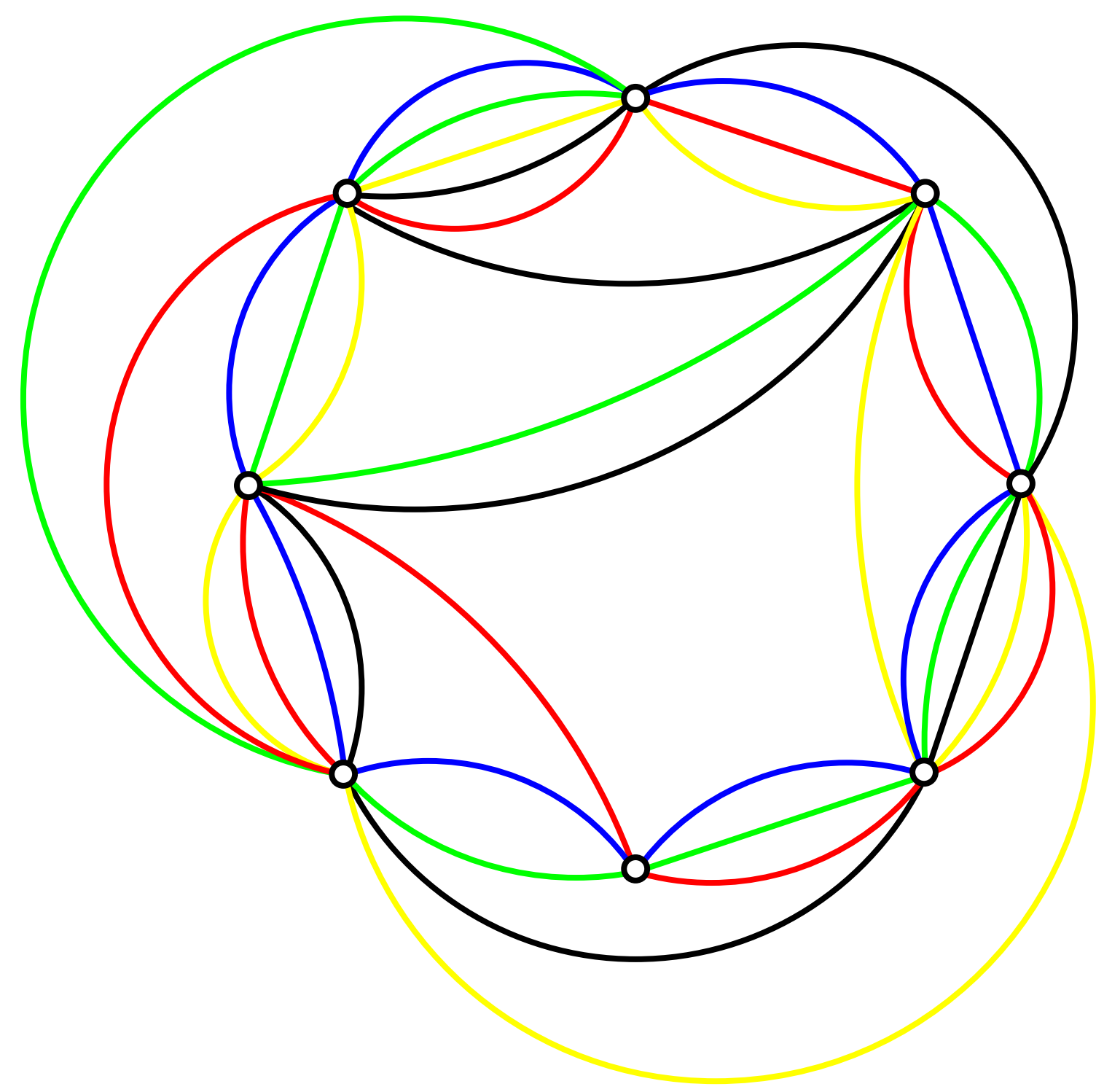

Figure 4: A 5-Venn diagram with 8 vertices. 


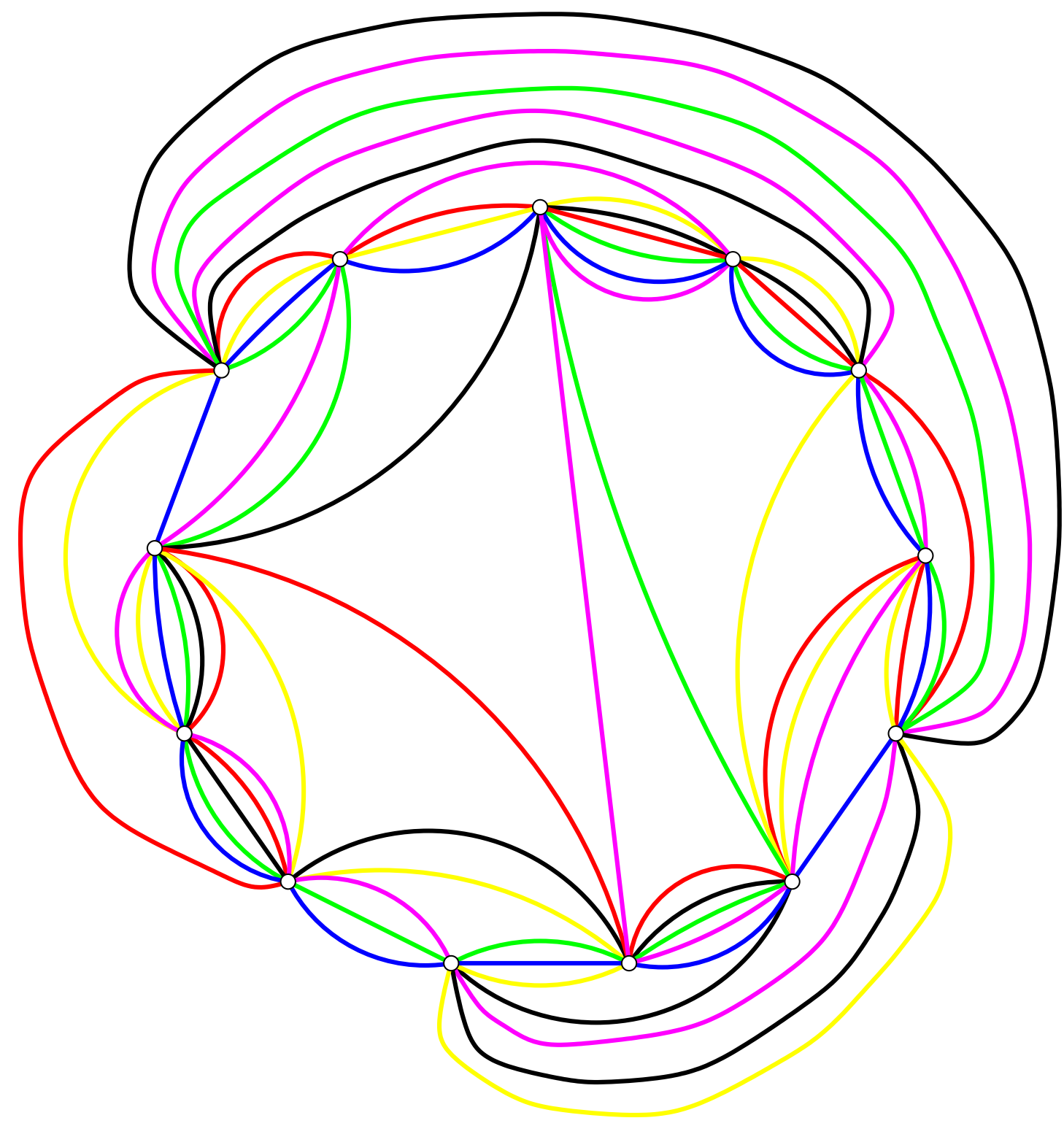

Figure 5: A 6-Venn diagram with 13 vertices. 


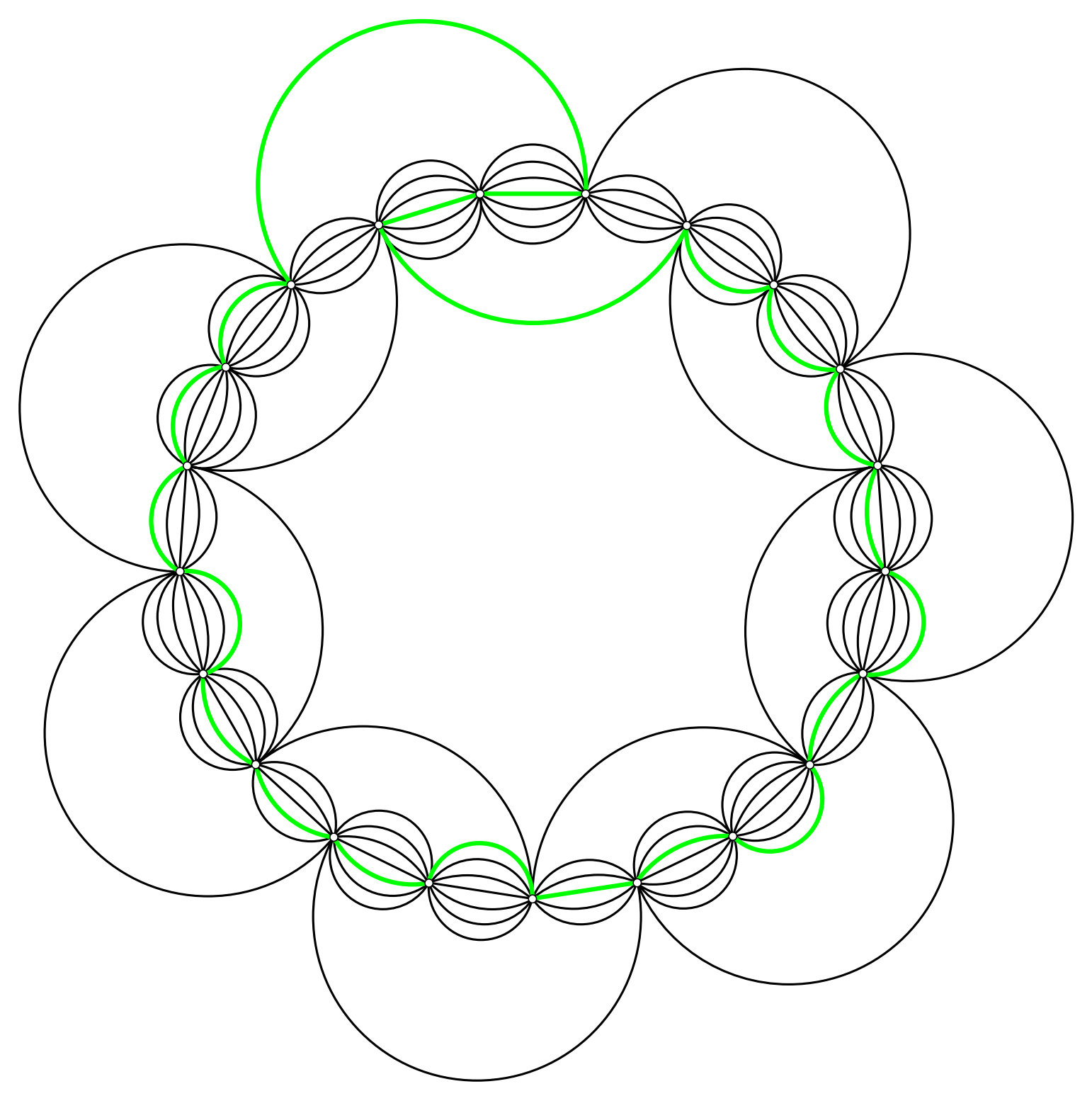

Figure 6: A 7-Venn diagram with 14 vertices. 
Proof: Choose any 2 consecutive (in a small circle around $d$ ) vertices $v$ and $w$ that are adjacent to $d$ in $R(P)$. Without loss of generality, let $v$ be a vertex of $P$ and $w$ a dual vertex in the region $S$ of $P$. Then $v$ is also contained on the facial cycle of $S$ and therefore is adjacent to $w$.

An interesting property of monotone Venn diagrams is that they can be peeled. For an $n$-Venn diagram $V$ and an integer $k \geq 1$, the $k$-peeled subgraph $V_{k}$ of $V$ is obtained by first removing all edges that border two regions in $V$ of weights less than $k$, and then removing all isolated vertices.

Lemma 3.3 A k-peeled subgraph $V_{k}$ of a monotone $n$-Venn $V$ contains every original region whose weight is at least $k$, and no bounded regions of weight less than $k$.

Proof (by induction on $k$ ): For the base case observe that $V_{1}$ is the same as $V$.

For $k \geq 1$, assume the statement is true. Consider $V_{k}$, the $k$-peeled graph of a monotone $n$-Venn diagram $V$, and its original dual graph $D(V)$.

Each dual vertex with weight $k$ is connected to at least one dual vertex of weight $k-1$, by the definition of a monotone Venn diagram. By the induction hypothesis, each dual vertex with weight $k$ is contained in a closed region of $V_{k}$, while each weight $k-1$ dual vertex is located in the unbounded region of $V_{k}$. By definition of the dual graph, there is an edge in the Venn diagram that corresponds to each dual graph edge between two dual vertices of weights $k-1$ and $k$. The removal of each of these Venn edges, peels $V_{k}$ and opens each $k$-region to the outer unbounded region.

None of the regions with weight greater than $k$ are affected. No $k$-region is left bounded in the peeled graph. Therefore the statement is true for $V_{k+1}$.

Using the same steps as in the construction of the radual graph of an $n$-Venn diagram, we construct the radual graph of a $k$-peeled graph of a monotone $n$-Venn diagram. Note that if we remove the dual vertex associated with the unbound region, we have a subgraph of the radual graph associated with the original monotone $n$-Venn diagram.

Theorem 3.1 For any radual graph $R(V)$, of a monotone $n$-Venn diagram $V$, and any $0<k<n$, there is a cycle of size $2\left(\begin{array}{l}n \\ k\end{array}\right)$ in $R(V)$, consisting of alternating Venn vertices and dual vertices with weight $k$.

Proof: Consider $V_{k}$, the $k$-peeled graph of an $n$-Venn diagram $V$. By Lemma 3.3, there are no regions with weight $k-1$ within $V_{k}$. Therefore, all weight $k-1$ regions of $V$ are part of the unbounded region in $V_{k}$. Since all the weight $k$ regions in $V_{k}$ must share an edge with regions with weight $k-1$, there are $\left(\begin{array}{l}n \\ k\end{array}\right)$ outer edges on $V_{k}$.

Now consider the radual graph, $R\left(V_{k}\right)$ : Let $d$ be the dual vertex in $R\left(V_{k}\right)$ of the unbounded region in $V_{k}$. By Lemma 3.1, $\operatorname{deg}(d)=2\left(\begin{array}{l}n \\ k\end{array}\right)$, and by Lemma 3.2, the vertices adjacent to $d$ form a cycle which alternates between Venn vertices and dual vertices with weight $k$. Since neither $d$ nor any of its edges are involved, this cycle is contained in the subgraph of $R(V)$. 
Let $M_{n}$ be the minimum number of vertices in a monotone $n$-Venn diagram. To prove that $M_{n}=\left(\begin{array}{c}n \\ \lfloor n / 2\rfloor\end{array}\right)$, we first show this is a lower bound, and then show that this value is attained by a certain sequence of Venn diagrams. We obtain the lower bound of $M_{n}$ from the number of $(n / 2)$-subsets of $\{1,2, \ldots, n\}$.

Theorem 3.2 If $n>1$, then

$$
M_{n} \geq\left(\begin{array}{c}
n \\
\lfloor n / 2\rfloor
\end{array}\right) .
$$

Proof: By Theorem 3.1, there exists a cycle on the radual graph of a monotone $n$ Venn of size $2\left(\begin{array}{l}n \\ k\end{array}\right)$, where $k=\lfloor n / 2\rfloor$. Since this cycle alternates between dual vertices and Venn vertices,

$$
M_{n} \geq\left(\begin{array}{c}
n \\
\lfloor n / 2\rfloor
\end{array}\right) .
$$

\subsection{A Straightened Venn Diagram}

Suppose that $V$ is an $n$-Venn diagram with a vertex $v$ such that $\operatorname{deg}(v)=2 n$. Let $v$ have a vertex traversal such that it is possible to split it into two copies where each copy is adjacent to $n$ distinct curves; i.e., where the vertex traversal consists of two contiguous subsequences, each containing $n$ curves. Imagine pulling the two copies of $v$ apart, horizontally stretching the rest of the curves so one of the curves $C$ becomes a straight line segment. Each of the curves and the intersections are stretched but do not change their original relationships. The resulting diagram represents a Venn diagram with $n$ simple curve segments beginning and ending at the two copies of $v$. The exterior region is now represented by the area above the curves and the interior region is represented by the region below the curves. We call this diagram a straightened representation of $V$.

Definition 3.1 We define an n-Straightened Venn Diagram, (n-SVD) as a straightened representation of an $n$-Venn diagram, $V_{n}$ with the following properties:

1. The curve $C_{n}$ is a horizontal line segment, beginning and ending on the two copies of vertex $v_{1}$, named $v_{1}^{L}$ and $v_{1}^{R}$.

2. All vertices of $V_{n}$ lie on $C_{n}$ and are numbered $v_{1}^{L}, v_{2}, \ldots, v_{m}, v_{1}^{R}$.

3. There are exactly $n$ vertices with degree $2 n$, including $v_{1}$ and $v_{2}$.

4. Any vertical line drawn through $C_{n}$ intersects each curve exactly once.

5. All non-adjacent vertices on $C_{n}$ are the endpoints of exactly 0 or 2 edges. 
Note that this diagram becomes a Venn diagram if we join the two copies of $v_{1}$ and make $C_{n}$ a circle.

Lemma 3.4 Any SVD represents a monotone Venn diagram.

Proof: By definition, the SVD represents a Venn diagram. It follows from Property 4 that the vertical line can be seen as a path through the directed dual graph, starting in the upper region, and ending in the lower.

Lemma 3.5 In an $n$-SVD, the number of curves intersecting at a vertex has the same parity as $n$.

Proof (by induction on $v_{k}$ ):

Let $h_{k}$ be the number of curves intersecting at a vertex $v_{k}$. Note that $h_{k}=$ $\operatorname{deg}\left(v_{k}\right) / 2$. Also, since an SVD is monotone, $h_{k}$ is the number of edges from $v_{i}$ to $v_{k}$, taken over all $i$ for which $1 \leq i<k \leq n$.

Base Case: By property 3 of Definition 3.1, $h_{1}=n$.

Inductive Step: Assume the statement is true for all $v_{k}$, where $k \geq 1$.

Let the number of edges from $v_{k}$ to $v_{k+1}$ be $c$. Let the number of edges from $v_{k}$ to $v_{l}$, where $l>k+1$, be $d$. Let the number of edges from $v_{j}$ to $v_{k+1}$, where $j<k$, be $g$. Then $h_{k}=c+d$, and $h_{k+1}=c+g$. By Property 5 of Definition 3.1, $d$ and $g$ are even. Then by the induction hypothesis, $c$ must have the same parity as $n$. Therefore, $h_{k+1}$ has the same parity as $n$.

Before we can construct an $(n+1)$-SVD from an $n$-SVD, we need a method to reduce the number of vertices on a Venn diagram. This method is defined in the next section.

\subsubsection{Vertex Compression}

We can compress 2 adjacent vertices $v$ and $w$ on a Venn diagram if they share exactly one common curve $C$. This is done by removing the edge $C(v w)$ and then mending the curve $C$ by merging $v$ and $w$. The process reduces the number of vertices by one, while maintaining the Venn diagram properties. All curves remain simple and closed and no regions have been created or destroyed.

We use this operation to prove the next theorem. An illustration of the proof can be found in Figure 7, for the case $n=4$.

Theorem 3.3 An $n$-SVD can be extended to an $(n+1)-S V D$.

Proof: Let $V_{n}$ be an $n$-SVD with $m$ vertices. Divide the plane into $m$ sections $P_{1}, P_{2}, \ldots, P_{m}$, each section delimited by two vertical lines through two consecutive vertices on $V_{n}$.

Step 1 : We draw a new curve $C_{n+1}$ beginning at $v_{1}^{\mathrm{L}}$, and ending on $v_{1}^{\mathrm{R}}$. In each $P_{i}$, we move up to the highest region that has not been previously visited, and sweep 


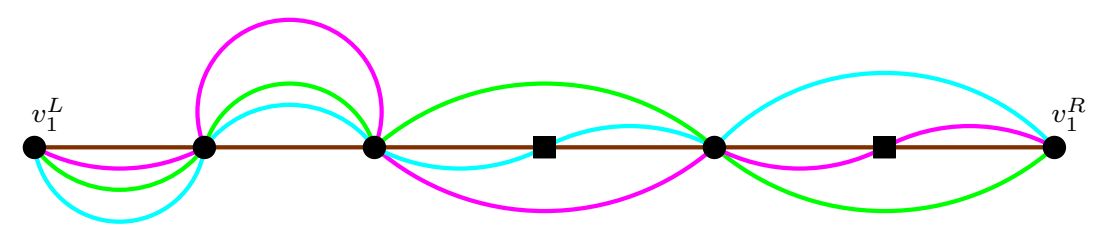

(a) The diagram $V_{4}$.

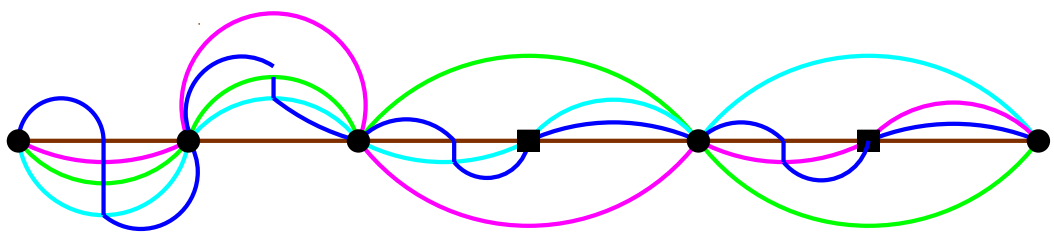

(b) The new curve moving through $V_{4}$.

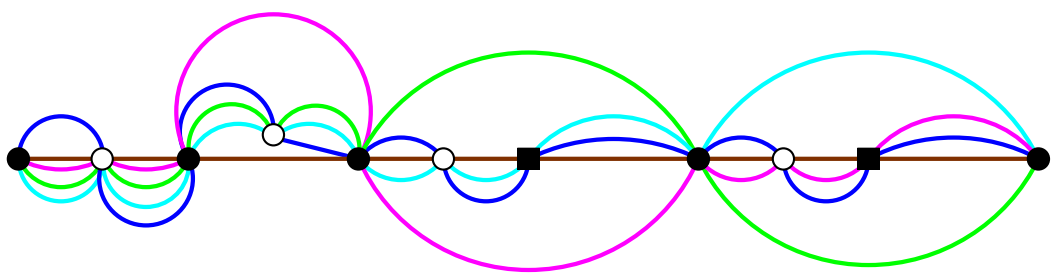

(c) Compression of vertices along the new curve.

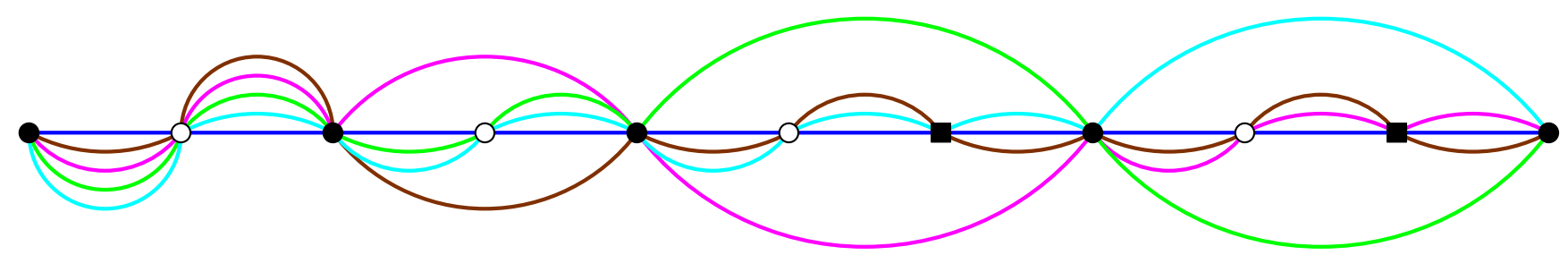

(d) Straighten the new curve to get $V_{5}$.

Figure 7: Constructing $V_{5}$ from $V_{4}$ 
downwards as far as possible through all non-visited regions, crossing curves as necessary. We exit each $P_{i}$ through the bordering vertex, and continue in this manner until we reach $v_{1}^{\mathrm{R}}$. Since $C_{n+1}$ passes through all $2^{n}$ regions, at the end of this step, we have a representation of a monotone Venn diagram which has been cut at $v_{1}$.

Step 2: The curve $C_{n+1}$, while in $P_{i}$, intersects $0 \leq r \leq n$ distinct curves on its downward sweep before it exits through the right vertex. For $r \geq 2$, we can apply the above compression operation $r-1$ times and create exactly one vertex from the previous $r$ vertices. This operation, performed similarly in each section, reduces the number of newly created vertices to no more than $2 \mathrm{~m}$.

Step 3: Straighten $C_{n+1}$.

The new diagram is an $(n+1)$-SVD because

1. The curve $C_{n+1}$ is the straightened horizontal line segment, beginning and ending on the two copies of $v_{1}$.

2. Since $C_{n+1}$ passes through all existing vertices, and creates the only new ones, all vertices lie on $C_{n+1}$, and are numbered $v_{1}^{\mathrm{L}}=w_{1}^{\mathrm{L}}, w_{2}, \ldots, w_{t}, w_{1}^{\mathrm{R}}=v_{1}^{\mathrm{R}}$, where $t \leq 2 m$.

3. $C_{n+1}$ crosses all existing curves in one downward sweep in the first section of $V_{n}$, between $v_{1}^{\mathrm{L}}$ and $v_{2}$, and these new vertices are compressed to form one vertex of degree $2(n+1)$. After that, $C_{n+1}$ does not venture into the upper or lower regions again, and therefore we cannot produce another compression involving all the curves. The curve $C_{n+1}$ passes through all existing vertices on $V_{n}$, so any vertices that had degree $2 n$ previously, will now have degree $2(n+1)$. The total number of vertices having degree $2(n+1)$ is $n+1$.

4. Since $C_{n+1}$ is a straight line, it can be intersected by a vertical line exactly once. The curves of $V_{n}$ continue to move left to right in the new diagram.

5. If vertices $u$ and $w$ are non-adjacent in $V_{n}$, then there are zero or two edges incident to both. If the number is two, then $C_{n+1}$ has already visited the regions above and below these edges, before passing through $u$. In either case, $C_{n+1}$ does not alter the number of edges incident to $u$ and $w$.

If the vertices are adjacent in $V_{n}$, then prior to passing through $u$, curve $C_{n+1}$ has either visited the regions above and below the outermost edges, or it has not. If it has not, then $C_{n+1}$ crosses all the edges, and $u$ and $w$ share no edges in the new diagram. If it has, then $C_{n+1}$ does not cross the upper and lower edges, and $u$ and $v$, if they are no longer adjacent, are the endpoints of exactly two edges.

If we use the same construction described in the proof of Theorem 3.3, for all values of $n$, we create a sequence $V_{2}, V_{3}, V_{4}, \ldots$ of SVDs that has very interesting properties. When discussing SVDs from now on, we specifically refer to this set.

The construction of the first two diagrams is described below. 
1. For $n=1$, the curve $C_{1}$ is a horizontal line segment which divides the plane into an upper and lower region.

2. For $n=2$, the curve $C_{2}$ starts at $C_{1}$ 's leftmost point, moves up to the upper region, crosses $C_{1}$ into the lower region and stops at $C_{2}$ 's rightmost point. No compression is necessary and $C_{2}$ becomes the new straight line segment.

The next four diagrams created by our construction are shown in Figure 8.

\subsection{Properties of SVDs}

\subsubsection{Structural Properties}

Let $V_{n}$ be a straightened $n$-Venn diagram, constructed as described in the previous section. Let $v_{i}$ be a vertex on $V_{n}$ that has degree $2 n$. And let $v_{j}$ be the next vertex to the right of $v_{i}$, which also has degree $2 n$. We will call the portion of $V_{n}$ which is contained between these 2 vertices a football, $F_{k}^{n}$, where $k$ is an index number of the football, as we count from left to right. By Definition 3.1, $1 \leq k \leq n$.

Each football has a boundary, which consists of the edges that border the outer and inner region. A boundary edge is sometimes referred to as the upper or lower boundary edge.

Note that due to the method of construction, $C_{n}$ splits $F_{1}^{n-1}$ into $F_{1}^{n}$ and $F_{2}^{n}$. For $k>1, C_{n}$ does not cross a boundary in $F_{1}^{n-1}$. These two facts mean that the modified $F_{k-1}^{n-1}$ in $V_{n-1}$ is re-indexed as $F_{k}^{n}$ in $V_{n}$.

Lemma 3.6 The topological structure of $F_{k}^{n}$, where $1 \leq k \leq n$, is covered by one of the following statements:

1. When $1 \leq k \leq 2$, it is a collection of $n$ labelled edges.

2. When $3 \leq k \leq n-1$, it is a boundary containing $F_{1}^{n-2} \cdots F_{k-1}^{n-2}$, as illustrated in Figure 9.

3. When $k=n$ it is a boundary containing $F_{1}^{n-2} \cdots F_{n-2}^{n-2}$.

Proof (by induction on $n$ ):

See Figure 8 for the base cases of $n=2$ and $n=3$.

Assume the statement is true for $F_{1}^{n-1}$. The curve $C_{n}$ passes through all curves in $F_{1}^{n-1}$, from the upper to lower region. After compression and straightening $C_{n}$, we produce $F_{1}^{n}$ and $F_{2}^{n}$, divided by the only new vertex. Thus statement 1 is proven.

Assume the lemma is true for $F_{k-1}^{n-1}$ and consider $3 \leq k \leq n-1$ : When constructing $F_{k}^{n}$ from $F_{k-1}^{n-1}$, the curve $C_{n}$ does not cross the boundary, since $k>2$. For $k=3$, the action of adding $C^{n}$ to $F_{2}^{n-1}$ creates a single vertex compressing $n-3$ labelled edges. When $C^{n}$ is straightened, the structure within the newly indexed $F_{3}^{n}$ 's boundary is identical to $F_{1}^{n-2} F_{2}^{n-2}$. 


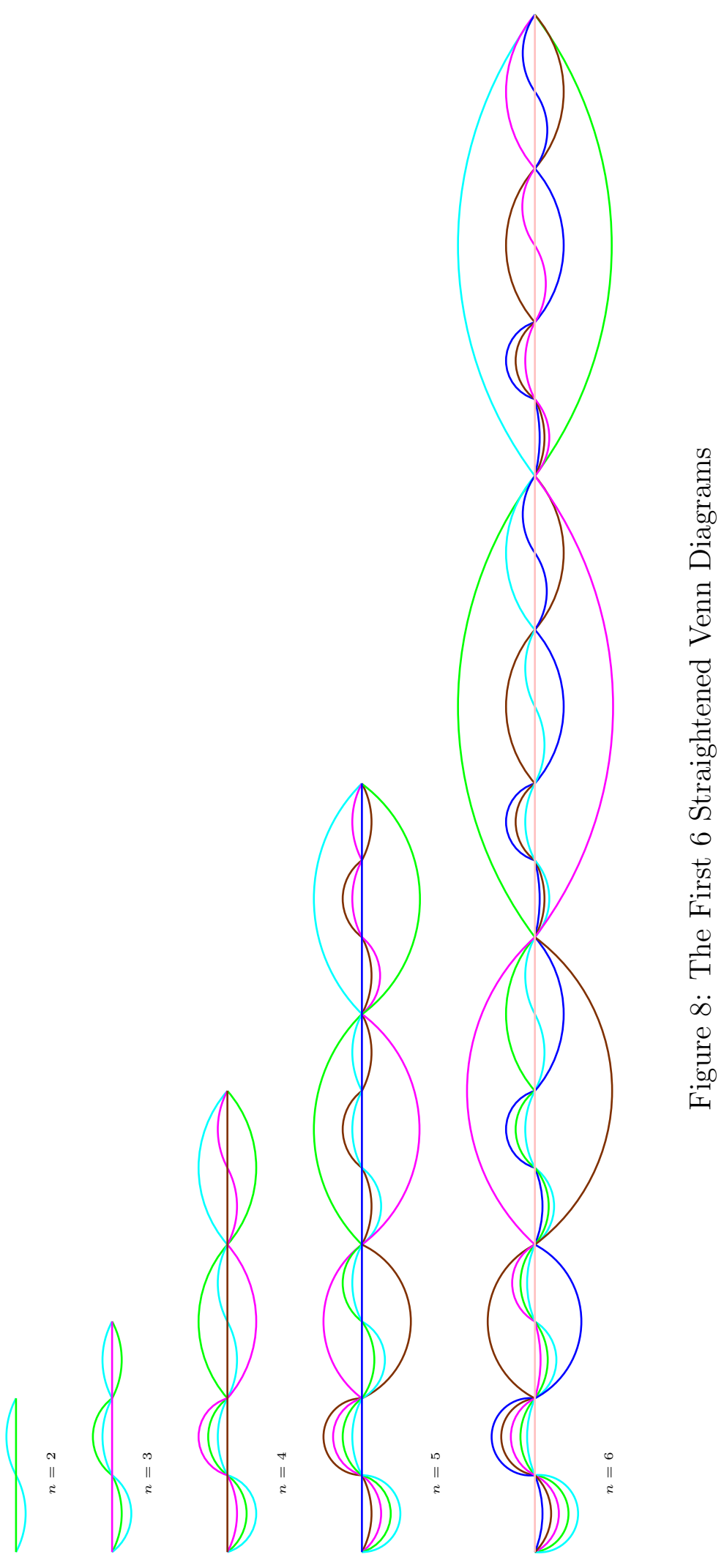




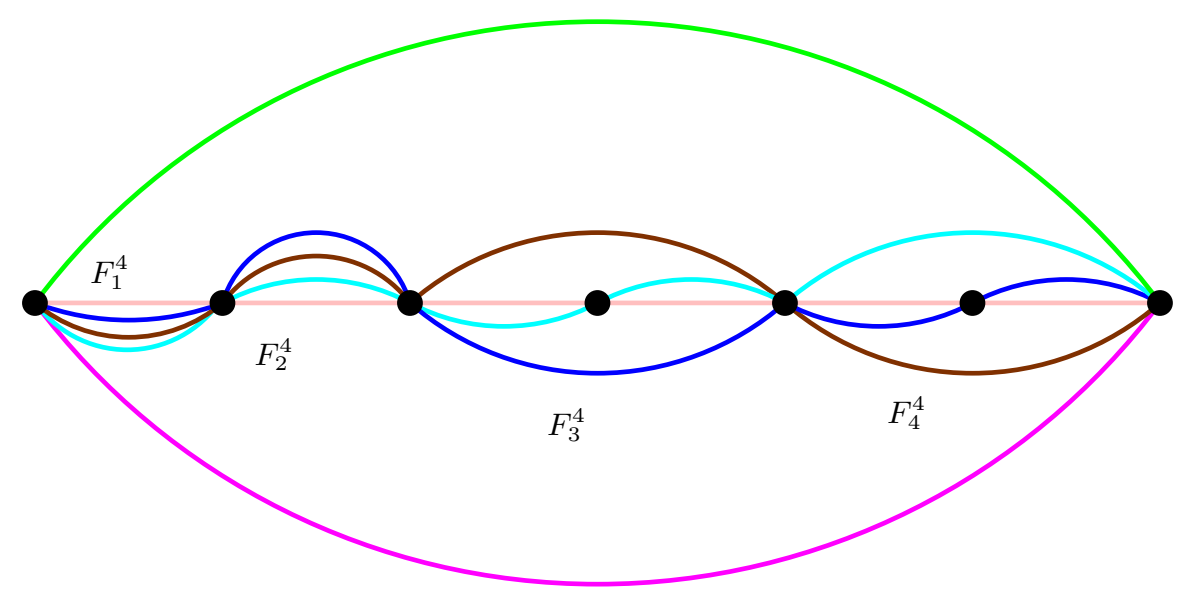

Figure 9: The Topological Structure of $F_{5}^{6}$

For $k>3$, by the induction hypothesis, $F_{k-1}^{n-1}$ 's boundary contains $F_{1}^{n-3} \cdots F_{k-2}^{n-3}$. For the special case of $k=n$, the boundary does not contain $F_{k-2}^{n-3}$, and for simplicity, this is assumed in all future statements concerning $F_{k}^{n}$. When $C_{n}$ is added to create $F_{k}^{n}$ from $F_{k-1}^{n-1}$, its action inside the boundary follows the same pattern as $C_{n-2}$ does in $F_{1}^{n-3} \cdots F_{k-2}^{n-3}$, when creating $V_{n-2}$ from $V_{n-3}$. The modified $F_{1}^{n-3} \cdots F_{k-2}^{n-3}$ in $V_{n-3}$ becomes $F_{1}^{n-2} \cdots F_{k-1}^{n-2}$, in $V_{n-2}$. The modified $F_{k-1}^{n-1}$, re-indexed as $F_{k}^{n}$ in $V_{n}$, contains $F_{1}^{n-2} \cdots F_{k-1}^{n-2}$. Thus statements 2 and 3 are proven.

\subsubsection{Curve Properties}

Another property of these straightened Venn diagrams is that within each football, the curve segment of $C_{i}$ has a predictable placement. It is clear from the construction that the curve segments in $F_{1}^{n}$ are the edges ordered $C_{n}, \ldots, C_{1}$ and the curve segments in $F_{2}^{n}$ are ordered $C_{n-1}, \ldots, C_{1}, C_{n}$.

Lemma 3.7 For $2 \leq k \leq n$, the curve segment of $C_{j}$ in $F_{k}^{n}$ is described by one of the following statements:

1. When $1 \leq j<n-k+1$, the segment is the same as its placement in $F_{1}^{n-2} \cdots F_{k-1}^{n-2}$.

2. When $j=n-k+1$, the segment is the upper boundary.

3. When $j=n-k+2$, the segment is the lower boundary.

4. When $n-k+2<j \leq n$, the segment replaces the segment of $C_{j-2}$ in $F_{1}^{n-2} \cdots F_{k-1}^{n-2}$.

Proof: Note that for simplicity, when $k=n$, we assume that references to $F_{1}^{n-2} \cdots F_{k-1}^{n-2}$ do not include $F_{k-1}^{n-2}$.

When $j=n, C_{j}$ is the straight line segment. It is the upper boundary of $F_{1}^{n}$, the lower boundary of $F_{2}^{n}$, and clearly replaces $C_{n-2}$ in all of $F_{3}^{n} \cdots F_{n}^{n}$. 
For $k=2$, the curves are $C_{n-1}, \ldots, C_{1}, C_{n}$, from upper to lower boundary, so statements 1, 2 and 3 are proven, and 4 is not applicable.

For $k>2$ and $j<n$, we use induction on $n$. See Figure 8 for the base cases of $n=2$ and $n=3$.

Inductive Step: Assume the statement is true for any $C_{j}$ in $F_{k}^{n-1}$.

Since $n-k+1=n-1-(k-1)+1$, and $n-k+2=n-1-(k-1)+2$, we use the induction hypothesis to claim that boundaries of $F_{k-1}^{n-1}$ remain the same boundaries when $F_{k}^{n}$ is created. Thus statements 2 and 3 are true for all $n$.

For non-boundary values of $j$, we invoke the induction hypothesis to claim that $C_{j}$ in $F_{k-1}^{n-1}$ is either the same as its placement, or is replacing $C_{j-2}$, in $F_{1}^{n-3} \cdots F_{k-2}^{n-3}$. $C_{n}$ acts on $F_{k-1}^{n-1}$ in the same manner as $C_{n-2}$ acts on $F_{1}^{n-3} \cdots F_{k-2}^{n-3}$, creating $F_{k}^{n}$ or $F_{1}^{n-2} \cdots F_{k-1}^{n-2}$ respectively. So $C_{j}$ in $F_{k}^{n}$ is either still the same as its placement, or is still replacing $C_{j-2}$, in $F_{1}^{n-2} \cdots F_{k-1}^{n-2}$. Thus statements 1 and 4 are true for all $n$.

\subsection{Counting the Vertices}

We have determined in the proof of Theorem 3.3, that the number of vertices of $V_{n}$ is no more than twice the number of vertices of $V_{n-1}$. In order to precisely determine the number of vertices, we need to subtract the number of times that $C_{n}$ passes through 2 existing vertices in $V_{n-1}$, without crossing an edge.

For $n>2$, we say $V_{n}$ has a singleton crossing, whenever it has a vertex of degree 4. During the construction of $V_{n+1}$, as $C_{n+1}$ exits this vertex, entering section $P_{i}$, it confines itself within the 2 curves and does not create a new vertex before exiting $P_{i}$. See the square vertices of Figure 7(a).

Lemma 3.8 If a singleton crossing occurs on $V_{n}$, then $n$ is even.

Proof: A singleton crossing means that 2 curves cross at one vertex. By Lemma 3.5, $n$ must be even.

Let $S(n, k)$ be the number of singleton crossings within the football $F_{k}^{n}$. For clarity, we define $S(2,1)=0$, and $S(2,2)=1$. We define $S(n)$ to be the total number of singleton crossings on an $n$-SVD.

Lemma 3.9 The number $S(n, k)$ is positive if and only if $n$ is even and $n / 2<k \leq n$.

Proof (by induction on $n$ ):

Obviously $k \leq n$, so we deal specifically with $n / 2<k$.

Base Case: For $n=2, S(2,2)=1$, and $S(2,1)=0$.

Inductive Step: Suppose it is true for $n-2$ and consider $F_{k}^{n}$.

Suppose $S(n, k)>0$ : We know that $n$ is even (by Lemma 3.8). Let $F_{i}^{n-2}$ be a football contained within the boundary of $F_{k}^{n}$, such that $S(n-2, i)>0$. Then by Lemma $3.61 \leq i \leq k-1$ and by the induction hypothesis

$$
i>\frac{n-2}{2}
$$


Therefore $n / 2-1<i$. $\leq k-1$, which implies that $n / 2<k$.

Suppose $n$ is even and $n / 2<k \leq n$. By Lemma 3.6, $F_{k}^{n}$ contains $F_{1}^{n-2} \cdots F_{k-1}^{n-2}$ within its boundary. Since $k-1>n / 2-1$, by the induction hypothesis $F_{k-1}^{n-2}$ must have a singleton crossing. Therefore, $S(n, k)>0$.

We now present three little corollaries concerning $S(n, k)$ :

Corollary 3.4 For all $n \geq 2$, we have $S(n, n)=S(n, n-1)$.

Proof: By Lemma 3.6, we know that the unlabeled $F_{n-1}^{n}$ is identical to the unlabeled $F_{n}^{n}$.

Corollary 3.5 If $n$ is even and $n \geq 2$, then $S(n, n / 2+1)=1$.

Proof (by induction on $n$ ):

Base Case:

$$
S(2,2)=1
$$

Inductive Step: Suppose it is true for $S(n-2, n / 2)$. Then

$$
\begin{aligned}
S(n, n / 2+1) & =\sum_{i=1}^{n / 2} S(n-2, i) \quad \text { (by Lemma 3.6) } \\
& =\sum_{i=1}^{n / 2-1} S(n-2, i)+S(n-2, n / 2) \\
& =0+1=1 \quad \text { (by Lemma } 3.9 \text { and the induction hypothesis). }
\end{aligned}
$$

Corollary 3.6 For all $1 \leq k \leq n$,

$$
S(n, k)=S(n, k-1)+S(n-2, k-1)
$$

\section{Proof:}

$$
\begin{aligned}
S(n, k) & =\sum_{i=1}^{k-1} S(n-2, i) \quad(\text { by Lemma 3.6) } \\
& =\sum_{i=1}^{k-2} S(n-2, i)+S(n-2, k-1) \\
& =S(n, k-1)+S(n-2, k-1) .
\end{aligned}
$$


Define $T(n, k)$ to be the number of well-formed parentheses strings of length $2 n$, which begin with exactly $k$ left parentheses. The following recurrence relation for $T(n, k)$ is proven in $\mathrm{Hu}$ and Ruskey [9].

$$
T(n, k)= \begin{cases}T(n, 2) & \text { if } k=1 \\ T(n, k+1)+T(n-1, k-1) & \text { if } 1<k<n \\ 1 & \text { if } k=n\end{cases}
$$

We use $T(n, k)$ to demonstrate a relationship between $S(n)$ and $C(n)$, the $n$th Catalan number. The Catalan numbers count the total number of well-formed parentheses strings of length $2 n$. Two equations for $C(n)$ are given below.

$$
\begin{aligned}
C(n) & =\frac{1}{n+1}\left(\begin{array}{c}
2 n \\
n
\end{array}\right) \\
& =\sum_{i=1}^{n} T(n, i) .
\end{aligned}
$$

Lemma 3.10 For an even integer $n>1$,

$$
S(n, k)=T(n / 2, n-k+1) .
$$

Proof (by induction on $\mathrm{k}$ ):

Base Case:

$$
S(n, n / 2+1)=1=T(n / 2, n / 2)
$$

Inductive Step: Assume the statement is true for all values less than $k$ :

$$
\begin{aligned}
S(n, k) & =S(n, k-1)+S(n-2, k-1) \quad(\text { by Corollary 3.6) } \\
& =T(n / 2, n-k+2)+T(n / 2-1, n-k) \\
& =T(n / 2, n-k+1) .
\end{aligned}
$$

And

$$
S(n, n)=S(n, n-1)=T(n / 2,2)=T(n / 2,1) .
$$

Corollary 3.7 For $n=2 m$, the number of singleton crossings $S(n)$ on $V_{n}$, is $C(m)$.

\section{Proof:}

$$
\begin{aligned}
C(m) & =\sum_{i=1}^{m} T(m, i) \\
& =\sum_{j=m+1}^{n} S(n, j) \quad \text { (by Lemma 3.10) } \\
& =S(n) .
\end{aligned}
$$




\subsubsection{Subtracting the Singleton Crossing from $2 M_{n}$}

We know from the proof of Theorem 3.3 and the previous section, that if $V n$ has $M_{n}$ vertices, then $V_{n+1}$ has $2 M_{n}-S(n)$ vertices.

Theorem 3.8 $\quad M_{n}=\left(\begin{array}{c}n \\ \lfloor n / 2\rfloor\end{array}\right)$.

Proof: Theorem 3.2 showed that $M_{n} \geq\left(\begin{array}{c}n \\ n / 2\end{array}\right)$. We proceed by induction on $n$.

Base Cases: Observe that $M_{2}=2$ and $M_{3}=3$, by Figure 8 .

Inductive Step: Let $n=2 m$, and assume that for all $k<n$,

$$
M_{k}=\left(\begin{array}{c}
k \\
\lfloor k / 2\rfloor
\end{array}\right) \text {. }
$$

Then for $n$, which is even,

$$
\begin{aligned}
M_{n} & \leq 2 M_{n-1}-S(n-1, k)=2 M_{n-1} \\
& =2\left(\begin{array}{c}
2 m-1 \\
m-1
\end{array}\right) \\
& =\frac{2(2 m-1) !}{(m-1) ! m !} \\
& =\frac{2 m(2 m-1) !}{m(m-1) ! m !} \\
& =\frac{2 m !}{m ! m !} \\
& =\left(\begin{array}{c}
2 m \\
m
\end{array}\right) \\
& =\left(\begin{array}{c}
n \\
\lfloor n / 2\rfloor
\end{array}\right) .
\end{aligned}
$$

And for $n+1$, which is odd,

$$
\begin{aligned}
M_{n+1} & \leq 2 M_{n}-S(n)=2 M_{n}-C(m) \\
& =2\left(\begin{array}{c}
2 m \\
m
\end{array}\right)-\frac{1}{m+1}\left(\begin{array}{c}
2 m \\
m
\end{array}\right) \\
& =\frac{2(m+1)-1}{m+1}\left(\begin{array}{c}
2 m \\
m
\end{array}\right) \\
& =\frac{(2 m+1)(2 m) !}{(m+1) m ! m !} \\
& =\frac{(2 m+1) !}{(m+1) ! m !} \\
& =\left(\begin{array}{c}
2 m+1 \\
m
\end{array}\right) \\
& =\left(\begin{array}{c}
n \\
\lfloor n / 2\rfloor
\end{array}\right) .
\end{aligned}
$$




\section{Acknowledgements}

We wish to express our thanks to Branko Grünbaum and Peter Hamburger for their helpful comments and discussion regarding this paper. Research supported in part by grants from the Natural Science and Engineering Research Council of Canada.

\section{References}

[1] B. Bultena, B. Grünbaum, and F. Ruskey, "Convex Drawings of Intersecting Families of Simple Closed Curves," submitted manuscript, 1998.

[2] K.B. Chilakamarri, P. Hamburger and R.E. Pippert, "Hamilton Cycles in Planar Graphs and Venn Diagrams," Journal of Combinatorial Theory, B 67 (1996) 296-303.

[3] K.B. Chilakamarri, P. Hamburger and R.E. Pippert, "Venn Diagrams and Planar Graphs," Geometriae Dedicata, 62 (1996) 73-91.

[4] A.W.F. Edwards, "Seven-set Venn diagrams with rotational and polar symmetry," Combinatorics, Probability, and Computing, 7 (1998) 149-152.

[5] A.W.F. Edwards, "Venn diagrams for many sets," Bulletin of the International Statistical Institute, 47th Session, Paris (1989) Contributed papers, Book 1, 311-312.

[6] A.W.F. Edwards, "Venn diagrams for many sets," New Scientist, 7 (January 1989) 51-56.

[7] B. Grünbaum, "Venn diagrams and Independent Families of Sets." Mathematics Magazine, 48 (Jan-Feb 1975) 12-23.

[8] P. Hamburger, "A Graph-theoretic approach to Geometry," manuscript, 1998.

[9] T.C. Hu and F. Ruskey, "Generating Binary Trees Lexicographically," SIAM J. Computing, 6 (1977) 745-758.

[10] F. Ruskey, "A Survey of Venn Diagrams," Electronic Journal of Combinatorics, 4 (1997) DS\#5. 